\title{
Enfermedad de Von Willebrand: Utilidad Diagnóstica de la Prueba de Tolerancia al Acido Acetilsalicílico
}

\author{
Dr. Ernesto Ríos L. ' Dra. Esperanza Marzouka B. ${ }^{2}$; T.M. Cecilia Cataldo T.',
}

\author{
Von Willebrand's Disease: \\ Diagnostic Usefulness of Acetil Salicilic Acid Tolerance Test
}

\begin{abstract}
Twenty nine patients with Yon Wiliebrand's disease (V W D) and 44 patients with norma] hemostasis, were studied.. In all of them template Ivy bleeding time (B.T.) was performed and repeated 2 hours after the ingestion of acetylsalicylic acid, a test known as ASA tolerance test (ASATT). In the patients with VWD, BT and ASATT were longer than in normal controls, but there were intermediate similat values in both groups. The higher limits of normality were defined as 7'45" for BT and 11' for ASATT. $72 \%$ of the patients with VWD had prolonged BT and $69 \%$ had prolonged ASATT; the joint analysis of both test increases their sensibility to $86 \%$ ASATT, in adittion to BT, helps in the diagnosis of mild bleeding disotders and if it is prolonged it must be comple mented with other specific tests to attain a definite diagnosis

(Key words: Acetylsaljcylic acid. Bleeding time. Toler ance test. Diagnosis of Von Willebrand's disease).
\end{abstract}

El estudio de un paciente con un aparente trastorno de la hemostasis es cada vez más importante sobre todo si hay que decidir una conducta quirúrgica. Día a día se han ido descubriendo métodos diagnósticos más precisos que han permitido definiciones más certeras de los trastornos de los factores plasmáticos de la coagulación, y de las alteraciones de la función plaquetaria. Con estos estudios también sé han identificado diferentes grados de intensidad de diversas enfermedades, sjendo precisamente el grupo de pacientes con manifestaciones leves el más díficil de abordar ${ }^{1}$.

La Enfermedad de Von Willebrand (E.V.W.), por ser la más frecuente en la población general, $y$ por tener expresiones que van desde manifestaciones mínimas a máximas, es la enfermedad más importante de sospechar y de diagnosticar. El tiempo de sangría de Ivy modificado (T.S.) es uno de los exámenes "in vivo" que tiene importancia para este diagnóstico, sin embargo a veces

1. Departamento de Pediatría, Hospital Luis Calvo Mackenna, Facultad de Medicina, Universidad de Chile.

2. Instituto de Salud Pública, Santiago. $y$, sobre todo, en los pacientes con manifestaciones leves puede ser normal.

Al ácido acetilsalicílico, al alterar la función plaquetaria $^{2}$ prolonga el TS. Existe una prueba de laboratorio denominada tolerancia al ácido acetilsalicílico (TAAS) que podría hacer aparente una alteración leve de la hemostasis primaria que no se manifieste espontảneamente en el TS usual, haciendo más sensible este examen de labora. torio.

La E.V.W. se diagnostica con certeza con complejos y costosos estudios, que incluyen el análisis del factor VIII (F VIII) en sus componentes coagulantes, antigénico y cofactor ristocetina, es por esto que si una prueba sencilla como la TAAS pudiera ayudar a descartar una E.V.W. leve o afirmar más una sospecha diagnóstica, tendría gran importancia en el manejo clínico habitual de estos pacientes.

Con estas motivaciones se evahuó la TAAS en un grupo de pacientes con E.V.W. y en un grupo de sujetos normales.

\section{MATERLAL Y METODOS}

Se estudiaron 29 pacientes pediátricos con antecedentes personales y familiares de hemorragias y E.V.W. comprobada. En todos se midió 
tiempo de sangría de lvy modificado ${ }^{3}, F$ VIIl coagulante $\left.(\mathrm{F} \mathrm{VIII})_{c}\right)^{4}, F$ VIII antigénico (F VIII Ag) por inmunoelectroforesis ${ }^{5}$ y F VIll cofactur ristocetina (F VIII CoR) por microaglutinación en placa ${ }^{6}$. Se consideraron valores normales de TS: < 7'45"; F VIII : 60 a $150 \%$; F VIII Ag: 0,62 a $1,5 \mathrm{U} / \mathrm{ml} ; \mathrm{F}$ VllI CoR: $>60 \%$. E] diagnóstico de E.V.W. se consideró positivo cuando existían dos o más exámenes alterados 0 un examen alterado más antecedentes de un familiar con E.V.W.?.

Además se estudió un grupo de 44 niños en quienes se realizaron exámenes de coagulación como requisito previo a una intervención quirúr. gica electiva. Este grupo estuvo formado por niños sanos, a excepción de la afección que motivó la indicación de cirugía electiva, sin antecedentés hemorrágicos personales ni familiares, sin antecedentes de ingestión de ningún medicamento en los 10 días preceḍentes al es. tudio. En ellos se efectuaron examenes de TS, tiempo de protrombina, tiempo de tromboplastjna parcial activada (TTPA) Factor VIIl coagulante y recuento de plaquetas en cámara, todos los cuales debian ser normates.

En los dos grupos de pacientes se estudiaron las diferencias entre el tiempo de sangría de Ivy modificado basal $y$ un nuevo TS en duplicado efectuado 2 horas después de administrar ácido acetilsalicílico $20 \mathrm{mg} \mathrm{x} \mathrm{kg} \mathrm{(TAAS)} \mathrm{por} \mathrm{vía} \mathrm{oral.}$

\section{RESULTADOS}

Los resultados del tiempo de sangría de Ivy modificado se observan en la Tabla 1:

\section{Table 1}

Tiempo de Sangría

\begin{tabular}{|c|c|c|c|}
\hline & & Percentil & Percentil \\
\hline & Media & 3 & 4 \\
\hline Grupo Control: $(n=44)$ & 5 '50" & $3^{\prime} 26^{\prime \prime}$ & $729 "$ \\
\hline Grupo E.V.W:: $(n=29)$ & $8 ' 16^{\prime \prime}$ & 4'51" & $13^{\prime} 34^{\prime \prime}$ \\
\hline
\end{tabular}

En el grupo control se obtuvieron valores similares a los de un estudio anterior ${ }^{6}$ que considera como límite superior de to normal en 7'45". Al aplicar este valor en los pacientes con E.V.W. 21 sujetos tuvieron TS prolongado (72\%). Sin embargo otros 8 niños, a pesar de tener una E.V.W. confirmada, tenían TS dentro de los límites normales.
Los resultados del promedio del duplicado de la TAAS se observan en la Tabla 2.

Tabb 2

Tiempo de Sangría después de la ingestión de Acido Acetil Sulicílico

$$
\text { Media } \frac{\text { Percentil }}{3} \frac{\text { Percentil }}{97}
$$

Grupo Control: $(\mathrm{n}=44) \quad 837^{\prime \prime} \quad 5^{\prime} 17^{\prime \prime} \quad 11^{\prime} 33^{\prime \prime}$

Grupo F.V.W.: $(n=29) \quad 12 ' 31^{\prime \prime} \quad 7^{\prime} 26^{\prime \prime} \quad 20^{\prime} 14^{\prime \prime}$

De esta forma se definjó como límite superior de lo normal de la TAAS 11 'y se consideró que un valor de 11 minutos es sugerente de una E.V.W. En el grupo con E.V.W. 20 pacientes (69\%) tuvieron TAAS 11; en contraste con un sólo sujeto del grupo control TAAS, lo que representa un $2 \%$ de falsos positivos.

También se miđió la diferencia en minutos que habia en cada paciente, entre el TS y TAAS. Los resultados se muestran en la Tabla 3.

Tabla 3

Madia $\frac{\text { Percentil }}{3} \frac{\text { Percentil }}{97}$

Grupo Control: $(n=44) \quad 2^{\prime} 26^{\prime \prime} \quad 54^{\prime \prime} \quad 4^{\prime} 44^{\prime \prime}$

Grupo E.V.W. (n=29) 4'23" l'02" 10'07"

Así se definió como diferencia máxima nor. mal entre el TS y TAAS 4'30" y se consideró que una diferencia de 4'30" es sugerente de E.V.W. En el grupo con E.V.W. 13 pacientes (45\%) tuvieron diferencia $>430^{\prime \prime}$; 'lo que ocurrió sólo en un caso del grupo control que corresponde al mismo enfermo con TAAS $>11^{\prime}$ y representa nuevamente un $2 \%$ de falsos positivos.

Después del análisis individual deI TS, TAAS y las diferencias entre ambos, se hizo un análisis de conjunto para ver en qué medida estos 3 elementos del tiempo de sangría ayudaban a sospechar E.V.W.: 21 pacientes $(72 \%)$ tenían TAAS $>11$, y/o diferencia TS - TAAS $>4$ '30' 0 ambos. 25 pacientes $(86 \%)$ tenían $\mathrm{TS}>75^{\prime \prime}$ y/o TAAS $>$ 11 'y/o diferencia entre los dos $>4$ '30": Sólo 4 pacientes con E.V.W. (14\%) tuvieron los tres factores del tiempo de sangría dentro del rango normal. 


\section{DISCUSION}

Con este estudio nos propusinos evaluar la utilidad de la TAAS en el diagnóstico de E.V.W. Por eso se analizaron pacientes con E.V.W. y pacientes normales muy bien definidos con otros exámęnes de hemostasia, en los cuales se evaluó la TAAS.

Los resultados demostraron algunos hechos conocidos, como que en la E.V.W. existe una prolongación de TS y que en ambos grupos la TAAS fue significativamente más larga que el $\mathrm{TS}^{3}$. El ácido acetilsalicilico produce una altera. ción de la función plaquetaria al inhibir la segunda fase de agregación. Este fenómeno ocurre por acetilación irreversible de la ciclo-oxigenasa, enzima plaquetaria encargada de conver. tir el ácido araquidónico en prostaglandinas y tromboxano, ambos fundamentales en la activa. ción plaquetaria ${ }^{2}$.

En un estudio reciente ${ }^{9}$, en pacientes pediátricos con síntomas hemorrágicos y TS prolongado, se diagnosticó E.V.W. en $77 \%$ de ellos, 10 que hace de ella el trastorno de este'tipo 'mas importante de reconocer. La E.V.W. tiene manifestaciones clinicas que varían desde minimas a máximas y su identificación requiere complejos exámenes de laboratorio. En general la E.V.W. muy sintomática hace evidente la necesidad del estudio y el laboratorio confirma el diagnóstico con facilidad. Por el contrario enfermos con sintomas leves, historia familiar normal y exámenes de coagulación para orientación normales (TS, TTPA, Protrombina y Recuento de Plaque. tas), plantean al médico la pregunta de si debe continuar con exámenes más complejos o desechar el diagnóstico de E.V.W. Es precisamente en esta situación donde la TAAS cumple su rol, porque es un examen fácil de efectuar si se establecen estándares adecuados a sus variables.

Las cifras normales de TAAS < 11' son similares a las encontradas por Stuart y col. ${ }^{10} \mathrm{de}$ $<10^{\prime} 30^{\prime \prime}$. Sin embargo, el aumento de sensibilidad para sospechar la E.V.W. que produce la TAAS, de $72 \%$ a $86 \%$ de los pacientes obtenido en este estudio, es diferente al encontrado por dicho autor que alcanzó de $36 \%$ a $93 \%$. Una explicacjón a este hecho, es que en el trabajo aludido, se estudió una población de pacientes menos definida, que tuvo TS normal en una alta proporción (49/71), y rangos de normalidad muy estrechos $\left(<5^{\prime} 30^{\prime \prime}\right)$.

El estudio de la enfermedad hemorrágica leve debe extenderse a alteraciones minimas de la función plaquetaria, que en el presente trabajo no se imcluyeron, pero Czapek $^{11}$ midió la TAAS en este tipo de pacientes, como examen de orientación, antes de efectuar estudjos de agregación plaquetaria.

\section{CONCLUSIONES}

La TAAS sumada con el TS de Ivy modificado es un examen sertillo de orientación, que debe ser efectuado en la evaluación de un paciente con enfermedad hemorrágica leve. Cuando se asocian el estudio de la TAAS y el TS de Ivy modificado, en conjunto, aumenta la capacidad para sospechar con acierto E.V.W. de $71 \%$ a $86 \%$. En pacientes con TAAS y TS de Ivy modificado prolongados deben efectuarse los estudios del factor VIII y de agregación plaquetaria para llegar al diagnóstico definitivo.

\section{RESUMEN}

Se estudiaron 29 pacientes con enfermedad de Von Willebrand (E.V.W.) comprobada y 44 pacientes sin trastornos de la hemostasis. En todos ellos se efectuó un tiempo de sangría de Ivy modificado (TS) basal y se repitió, 2 horas después de la administración de $20 \mathrm{mg} x \mathrm{Kg}$ de ácido acetilsalicilico, esto constituye la prueba de tolerancia al ácido acetilsalicílico (TAAS). Se observó que los pacientes con E.V.W. tienen un TS y una TAAS, más prolongados que los controles normales. Sin embargo, hay valores intermedios similares en ambos grupos. Se definió como límite máximo de lo normal para TS 7'45" y para la TAAS 11'. En $72 \%$ de los pacientes con E.V.W. se encontró un TS prolongado y en $69 \%$ la TAAS estaba prolongada. Al analizar ambos resultados combinados la sensibilidad del método aumentổ a $86 \%$.

Se concluye que la TAAS agregada al TS de Ivy modificado, ayuda en la sospecha diagnóstica del paciente con enfermedad hemorrágica leve $y$ que si se encuentran prolongados deben efectuarse otros exámenes específicos para llegar al diagnóstico definitivo.

\section{REFERENCIAS}

1. Bachmann $F_{\text {.: }}$ Diagnostic approach to mild bleeding disorders Serninar. Hematol. 17: 292, 1980.

2. Roth G.J., Stanford N. and Majerus P.W.: Acetylation of prostaglandin synthetase by aspirin. Proc. Nat. Acad. Sci. USA. 72: 3073, 1975.

3. Mielke CH., Xaneshiro M.M., Waher I.A., Weiner J.M. and Ropaport S.I.: The standardized normal Ivy blexding time and its prolongation by aspirin. Blood 34: 204, 1969. 
4. Langdell R.D., Wagner R.H. and Brinckhous K.: Effect of antihemophilic factor on one stage clotting test. A presumptive test for hemophilia and a simple one stage antihemophilic factor assay procedure. J. Lab. Clin. Med. 41: 637, 1953.

5. Zimmeman T.S., Hoyer L.W., Dickson L, and Euingron T.S.: Determination of the Von Willebrand's disease antigen (Factor VIII related antigen) in plasma by quantitative inmmunoelectrophoresis. J. Lab. Clin. Med. 86: 152, 1975.

6. Reisner H.M. Katz H.J., Goldin L.R., Barrow E.S. and Graham J.B.: Use of a simple visual assay of $V_{\text {on }}$ Willebrand's factor for diagnosis and carrier identification Br. J. Haematol 40: 339, 1978.

7. Mezzano $D$, Aranda $E$, Grebe $G$., Legües M.E., Rodriguez S., Marzouka E, y Rios E.: Dingnóstico de kboratorio de la Enfermedad de Von Willebrand en 52 paciente. Rev. Med. Chile. 111: 1139, 1983.

8. Rios E., Pinochet $M$. y Mondujano M.: Tjempo de sangría de Ivy roodificado: Valores normales en edad pediátrica. Rev. Chil. Pediatr. 53: 552, 1982.

9. Marzouka E., Rios E., Mezzano D., Schuh W., Aranda $E$., Catoldo C. and Legües M.E.: El paciente con hemorragia y tiempo de sangia prokngado: Clasificación y diagnóstico diferencial. Rev. Chit. Pediatr. 56: 167, 1985.

10. Stuart M.J., Miller M.L., Davey F,R. ana' Wolk J.A.; The postaspirin bleeding time: a screening test for evaluating haemostatic disorders Br. J. Haematol. 43: 649, 1979.

11. Crapek E.E., Deykin D., Salzman E., Lian E.C., Hellerstein L.J. and Rosoff C.B.: Intermediate syndrome of platelet dysfunction. Blood 52: 103, 1978. 